\section{UAV and Piecewise Convex Approximation Assisted Localization With Unknown Path Loss Exponents}

\author{
Boda Liu ${ }^{(D)}$, Xu Zhu ${ }^{(D)}$, Senior Member, IEEE, \\ Yufei Jiang ${ }^{\mathbb{D}}$, Member, IEEE, Zhongxiang Wei ${ }^{\mathbb{D}}$, Member, IEEE, \\ and Yi Huang, Senior Member, IEEE
}

\begin{abstract}
In this correspondence, we investigate unmanned aerial vehicle (UAV)-base stations (BSs) assisted and received signal strength (RSS) based mobile station (MS) localization. A practical air-to-ground path loss model is utilized, where the path loss exponent (PLE) varies with the elevation angle and altitude of UAV, and the accurate PLE estimate is often difficult to obtain. With unknown and unequal PLEs for different UAVs, the UAVs assisted localization problem becomes nonlinear and non-convex, which cannot be solved by the existing methods. We propose a piecewise convex approximation aided localization (PCAL) approach to convert the localization problem into linear and convex, without requiring the knowledge of PLE. The proposed PCAL approach with unknown and unequal PLEs achieves much higher accuracy than the existing methods which require perfectly known and equal PLE, due to its higher robustness against shadowing. In addition, a grid search aided ambiguity elimination (GSAE) method, which is more effective than the state-of-the-art methods, is proposed to determine the final MS localization estimate based on multiple tentative estimates derived from PCAL. The effectiveness of PCAL is also verified by the Cramer-Rao lower bound (CRLB) derived.
\end{abstract}

Index Terms-Unmanned aerial vehicle, localization, received signal strength, path loss exponent, piecewise convex approximation.

\section{INTRODUCTION}

Unmanned aerial vehicle (UAV) mounted base station (BS) is regarded as a promising complementary solution for $5 \mathrm{G}$ in emergency cases like network damage and congestion [1]. UAV-BS provides better location based services (LCS) than ground BS due to higher probability of line of sight [2].

\section{A. Related Work}

Received signal strength (RSS) based localization of mobile station (MS) [3] has been widely used due to its low cost and low complexity. The terrestrial localization approaches in [4]-[11] utilized RSS

B. Liu and Y. Huang are with the Department of Electrical Engineering and Electronics, The University of Liverpool, L69 3GJ Liverpool, U.K. (e-mail: hsbliu6@liverpool.ac.uk; huangyi@ liverpool.ac.uk).

$\mathrm{X}$. Zhu is with the Department of Electrical Engineering and Electronics, The University of Liverpool, L69 3GJ Liverpool, U.K., and also with the School of Electronic and Information Engineering, Harbin Institute of Technology, Shenzhen 518055, China (e-mail: xuzhu@liverpool.ac.uk).

Y. Jiang is with the School of Electronic and Information Engineering, Harbin Institute of Technology, Shenzhen 518055, China (e-mail: jiangyufei@hit.edu.cn).

Z. Wei is with the School of Electronics and Electrical Engineering, University College London, WC1E 7JE London, U.K. (e-mail: zhongxiang.wei@ucl.ac.uk.). based exponential-like ranging function, is obtained by calculating the logarithm of RSS, to estimate mobile station (MS) location. Localization of MS with unknown transmit power was investigated in [4]. Noncooperative and cooperative localization approaches with unknown transmit power and unknown path loss exponent (PLE) were studied in [5]. In [6], least-square absolute error of ranging was minimized for localization. In [7], both RSS and differential RSS based localization methods were proposed, with anchor coordinates uncertainties and imperfect knowledge of PLE. A multilateration method, referred to as bias-compensated weighted least-square (bcWLS), was proposed in [8], where the perturbations in both RSS measurement error and anchor uncertainties are mitigated. In [9], geometric parameters were proposed for anchor deployment in localization. A particle filter based on data fusion was proposed in [10]. Multi-dimensional scaling techniques were proposed in [11] to build the connectivity map of deployed sensors.

The ranging function in RSS based localization is highly dependent on path loss model. In [4]-[11], a two-dimensional path loss model was utilized for terrestrial localization. In [12] and [13], a three-dimensional terrestrial path loss model was utilized for UAV assisted and RSS based localization. However, the UAV air-to-ground path loss model has been reported to be highly dependent on the elevation angle of the path and UAV's altitude [14]-[18], which was not considered by most existing work. In [18], a localization approach for elevation angle dependent path loss (EAPL) model was proposed. However, perfectly known and equal PLEs were assumed for all UAVs, which is unpractical. With unknown and unequal PLEs, the RSS based localization problem becomes nonlinear and non-convex, which cannot be solved by the existing approaches. In [19], the Levenberg-Marquardt algorithm (L-M) was proposed to solve the nonlinear problem with unknown and unequal PLEs, whose accuracy is largely subject to measurement errors, due to the potential inappropriate damping factor employed by L-M and unreliable initialization of PLEs.

Moreover, the effect of airframe shadowing (AS) due to fixedwings UAV [2] could cause signal attenuation of up to $35 \mathrm{~dB}$ [17]. Therefore, it is necessary to consider the effect of AS on localization when fixed-wings UAVs are employed, which is absent in the existing work, e.g., [20].

\section{B. Main Contributions}

Motivated by the above open issues, we propose a fixed-wings UAV-BSs aided and RSS based localization technique with unknown and unequal PLEs. Our work is different in the following aspects:

1) The nonlinear and non-convex RSS based localization problem with unknown and unequal PLEs is solved by a piecewise convex approximation aided localization (PCAL) scheme, with two-step approximations: (a) convert the problem to a nonlinear convex problem through piecewise convex approximation and curve fitting; (b) convert the resulting nonlinear convex problem to a linear convex problem through Taylor's series expansion (TSE) approximation. Unlike the TSE only based methods in [4]-[8], PCAL does not require the PLEs associated with different UAVs to be perfectly known and equal to each other, and therefore it is more practical.

2) Thanks to its robustness against shadowing, especially AS, the proposed PCAL approach with unknown and unequal PLEs achieves higher accuracy than the approaches [6]-[8] with equal 


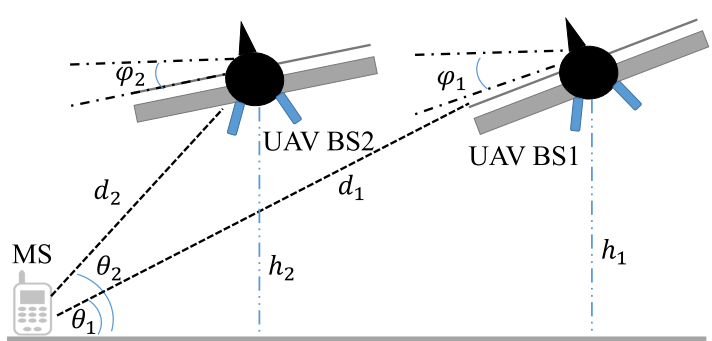

Fig. 1. Diagram of one MS located by multiple fixed-wing UAV-BSs.

and perfectly known PLEs, as well as the approach in [19] with unknown and unequal PLEs. This is because the power-form ranging function via piecewise convex approximation and TSE has much lower variance than the exponential-form ranging functions in [6]-[19]. To the best of our knowledge, this is also the first work to investigate the impact of AS on UAV assisted and RSS based localization. The Cramer-Rao lower bound (CRLB) on localization error is derived to verify the effectiveness of PCAL.

3) With $N$ UAV-BSs to locate one MS, PCAL produces $2^{N}$ objective functions and tentative estimates. A grid search assisted ambiguity elimination (GSAE) approach is proposed to obtain the final estimate of MS location by taking an average of the tentative estimates selected via grid search. GSAE is more effective than the piecewise-linear minimization (PLM) method [21] that selects just the tentative estimate with the minimum error between the estimated distance and the measured distance. The performance of PCAL-GSAE approaches the CRLB derived.

\section{System Model AND PROBlem Formulation}

We consider the localization of an MS assisted by $N$ UAV-BSs. Assume that the MS with the coordinate vector $\mathbf{v}=[x, y, z]^{T}$, is detected and located by $N$ UAV-BSs at a time instant (Fig. 1). Each UAV-BS supported cell is of horizontal radius $R$, and the accurate coordinates of the $i$-th UAV-BS is $\mathbf{w}_{i}=\left[x_{i}, y_{i}, z_{i}\right]^{T}, i=1,2, \ldots, N$. The elevation angle of the $i$-th UAV-BS is $\theta_{i}$, and the UAV roll angle is $\varphi_{i} \cdot \varphi_{i}$ and UAV's altitude $h_{i}$ are available at the UAV control system and barometer/GPS. The localization problem is

$$
\text { (P1) } \min _{\mathbf{v}} \sum_{i=1}^{N}\left(\widehat{P L}_{i}-\widetilde{P L}_{i}\right)^{2}
$$

where $\widehat{P L}_{i}$ and $\widetilde{P L}_{i}$ are the estimated and measured path loss at the $i$-th UAV-BS, respectively.

Considering the effect of AS [17] on path loss, the sum of lognormal random term of AS and the elevation angle dependent terrestrial shadowing (TS) [18] between MS and the $i$-th UAV-BS is denoted by $X_{S, i}$, where $X_{S, i} \sim \mathcal{N}\left(0, \sigma_{R S S, i}^{2}\right)$, and $\sigma_{R S S, i}^{2}=\sigma_{T S, i}^{2}+\sigma_{A S, i}^{2}$, where $\sigma_{T S, i}$ and $\sigma_{A S, i}$ are standard deviation of TS and AS, respectively. With fading smoothed out, the combined path loss and shadowing is expressed as

$$
P L_{i}=P t[\mathrm{dBm}]-P r_{i}[\mathrm{dBm}]+S_{0, i}+X_{S, i}
$$

where $S_{0, i}$ is a constant pre-determined by the roll angle $\varphi_{i}$. Evidently, $\widetilde{P L}_{i}=P t[\mathrm{dBm}]-P r_{i}[\mathrm{dBm}]$, where $P t$ is the transmit power, and $\mathrm{Pr}_{i}$ is the receive signal power at the $i$-th UAV.

The EAPL of the $i$-th UAV is calculated as [18]

$$
\widehat{P L}_{i}=10 \eta_{i} \log _{10}\left(d_{i}\right)
$$

$$
\eta_{i}=\frac{a_{1}}{1+a_{0} e^{-b_{0} \theta_{i}}}+b_{1}
$$

where $a_{0}, a_{1}, b_{0}, b_{1}$ are the environmental related parameters, and $\theta_{i}=$ $\arcsin \left(h_{i} / d_{i}\right)$ is the elevation angle of the $i$-th UAV, with $h_{i}$ being altitude of the $i$-th $\mathrm{UAV}$, and $d_{i}=\left\|\mathbf{v}-\mathbf{w}_{i}\right\|$ is given as the distance from the $i$-th UAV-BS to MS. Assume that the perfect knowledge of UAV's altitude $h_{i}$ is known, the EAPL in (3) is a function of single variable $d_{i}$.

\section{UAV Assisted AND PIECEWISE CONVEX APPROXIMATION Aided LOCALIZATION}

It is obvious that $(P 1)$ is a nonlinear and non-convex optimization problem. In Subsection III-A, we convert $(P 1)$ into a convex problem via piecewise convex approximation and curve fitting, and then into a linear problem via TSE. The approximation processes yield $2^{N}$ tentative estimates. The estimation ambiguity is eliminated by the GSAE approach in Subsection III-B. The CRLB of PCAL is derived in Subsection III-C.

\section{A. Piecewise Convex Approximation Aided Localization}

1) Transformation to a Convex Problem via Piecewise Convex Approximation and Curve Fitting: As EAPL is close to a sigmoid curve [18], it is partitioned into two sub-functions within the propagation distance of interest through piecewise convex approximation [22]. The transition point on EAPL of the $i$-th UAV is at distance $d_{i}=d_{t, i}$, which is selected as either a global maxima of the first derivative of EAPL (suitable for model in [18]) or a global minima of the EAPL function (suitable for model in [16]). Assume that the EAPL of the $i$-th UAV is partitioned into a piecewise convex function of two sub-functions in the intervals of $\left[d_{\text {lower }, i}, d_{t, i}\right]$ and $\left[d_{t, i}, d_{\text {upper }, i}\right]$, respectively, where $d_{\text {lower }, i}$ and $d_{\text {upper }, i}$ are the lower and upper bounds of the propagation distance of interest. Assisted by curve fitting, all the sub-functions are approximated to power functions as

$$
\widehat{P L}_{i, g_{i}} \approx A_{i, g_{i}} d_{i}^{B_{i, g_{i}}}+C_{i, g_{i}}
$$

where $A_{i, g_{i}}, B_{i, g_{i}}$, and $C_{i, g_{i}}$ are the fitted parameters of each subfunction, and $g_{i}=0$ or 1 indicates the first or second sub-function, respectively. The approximated path loss is converted to an explicit power-like ranging function as

$$
d_{i}=\left(1-g_{i}\right) d_{i, 0}+g_{i} d_{i, 1}
$$

where $d_{i, g_{i}}=\left(\left(\widehat{P L}_{i, g_{i}}-C_{i, g_{i}}\right) / A_{i, g_{i}}\right)^{1 / B_{i, g_{i}}}$. Round $\widetilde{P L_{i}}$ to the interval $\left[P L\left(d_{\text {lower }, i}\right), P L\left(d_{\text {upper }, i}\right)\right]$, in case of complex distance estimation caused by significant shadowing. Since $\widehat{P L}$ corresponds to two sub-functions shown by (5), and two distances, $d_{i, 0}$ and $d_{i, 1}$. If one MS is detected by $N$ UAV-BSs, there are total $2^{N}$ combinations of (5), and $2^{N}$ tentative estimates of MS localization, $\mathbf{v}_{j}, j=1,2, \ldots, 2^{N}$. Substituting (5) into (1) yields $(P 1)$ for one of the $2^{N}$ estimates as

$$
(P 2) \min _{\mathbf{v}, \mathbf{d}} \sum_{i=1}^{N}\left(A_{i, g_{i}} d_{i}^{B_{i, g_{i}}}+\beta_{i, g_{i}}-\widetilde{P L}_{i}\right)^{2}
$$

where $\beta_{i, g_{i}}=S_{0, i}+C_{i, g_{i}}$.

2) Transformation to a Linear Problem via Taylor's Series Expansion: The above nonlinear convex problem can be solved by maximum likelihood (ML) estimator. However, it usually requires an accurate initial guess to achieve the global optimal point rather than the local optimal point. To overcome the shortcoming of ML estimator, (7) can be rewritten as a linear convex optimization problem. If $S_{0, i}$ is 
known, the distance estimation of either interval in (6) can be further approximated to linear ranging function through the first-order TSE

$$
d_{i, g_{i}}=\left(\frac{\widetilde{P L_{i}}-\beta_{i, g_{i}}+X_{S, i}}{A_{i, g_{i}}}\right)^{\frac{1}{\bar{B}_{i, g_{i}}}} \approx \alpha_{i, g_{i}}+n_{i, g_{i}}
$$

Taking the square of both left-hand and right-hand sides of (8) yields $d_{i, g_{i}}^{2}-2 \alpha_{i, g_{i}} d_{i, g_{i}}+\alpha_{i, g_{i}}^{2}=n_{i, g_{i}}^{2}$, where $\alpha_{i, g_{i}}=$ $\left[\left(\widetilde{P L_{i}}-\beta_{i, g_{i}}\right) / A_{i, g_{i}}\right]^{1 / B_{i, g_{i}}}$, and $n_{i, g_{i}}^{2}=X_{S, i}^{2} /\left(A_{i, g_{i}}^{2} B_{i, g_{i}}^{2}\right)\left(\widetilde{P L_{i}}\right.$ $\left.\left.-\beta_{i, g_{i}}\right) / A_{i, g_{i}}\right)^{2 / B_{i, g_{i}}-2}$ is the mean square error (MSE) of ranging, and variance of $n_{i}$ is $\sigma_{i, g_{i}}^{2}=\sigma_{R S S, i}^{2}\left[\left(\widetilde{P L_{i}}-\beta_{i, g_{i}}\right) / A_{i, g_{i}}\right]^{2 / B_{i, g_{i}}-2} /$ $\left(A_{i, g_{i}} B_{i, g_{i}}\right)^{2}$. The MSE is smaller than those in [6]-[8] due to the two-step approximations. For the scenario with MS in the cell of radius $R=2000 \mathrm{~m}, h_{i}=1000 \mathrm{~m}$, and environmental data for urban area is $a_{0}=45, a_{1}=-1.5, b_{0}=10, b_{1}=3.5$, the minimum standard deviation in (8) $\sigma_{i, g_{i}} \approx 30 \sigma_{R S S, i}$, is smaller than that in [6]-[8] (about $\left.115 \sigma_{R S S, i}\right)$. Thus, $(P 3)$ is further converted to a semi-definite programming $(\mathrm{SDP})$ problem:

$$
\begin{aligned}
& (P 3) \min _{\mathbf{v}, \mathbf{d}, \mathbf{D}, \mathbf{z}} \sum_{i=1}^{N} p_{i, g_{i}}\left(D_{i, g_{i}}-2 \alpha_{i, g_{i}} d_{i, g_{i}}\right) \\
& \text { s.t. } \\
& D_{i}=\left[\begin{array}{l}
\mathbf{w}_{\mathbf{i}} \\
-1
\end{array}\right]^{T}\left[\begin{array}{ll}
\mathbf{I}_{3} & \mathbf{v} \\
\mathbf{v}^{\mathrm{T}} & \mathbf{Z}
\end{array}\right]\left[\begin{array}{l}
\mathbf{w}_{\mathbf{i}} \\
-1
\end{array}\right] \\
& D_{i, g_{i}} \geq d_{i, g_{i}}^{2} \\
& d_{\text {lower }, i} \leq d_{i, g_{i}} \leq d_{t, i} \text { or } d_{t, i} \leq d_{i, g_{i}} \leq d_{\text {upper }, i} \\
& {\left[\begin{array}{ll}
\mathbf{I}_{\mathbf{3}} & \mathbf{v} \\
\mathbf{v}^{T} & \mathrm{Z}
\end{array}\right] \geq \mathbf{0}}
\end{aligned}
$$

where $p_{i, g_{i}}=1 / \sigma_{i, g_{i}}^{2}$ is the weight of objective function, and $\mathbf{I}_{3}$ is $3 \times 3$ identity matrix, and $\mathrm{Z} \geq \mathbf{v}^{T} \mathbf{v}$ is the auxiliary variable. $(P 3)$ can be solved by interior-point method.

\section{B. Elimination of Estimation Ambiguity}

The proposed PCAL approach benefits from the low MSE of ranging. However, estimation ambiguity introduced by piecewise functions brings extra estimation error. It is eliminated by the GSAE approach: first finding a reference point $\mathbf{v}_{G S}$ through solving (7) by grid search, and then taking an average of the coordinates of the $M=2^{N}-N$ tentative estimates closest to $\mathbf{v}_{G S}$, which forms the set $\Lambda$.

$$
\overline{\mathbf{v}}=\frac{1}{M} \sum_{j \in \Lambda} \mathbf{v}_{j}
$$

The overall algorithm of PCAL alongside GSAE is summarized in Algorithm 1. The proposed single time localization approach can be easily extended to continuous localization by utilizing a filter such as particle filter [10].

The proposed scheme can be extended to a multi-MS scenario. The number of simultaneously located MSs is limited by the number of resolvable resource blocks (RBs) within a cell at each transmission time interval, and the number of MSs requesting the same quality of LCS [24]. For example, within a cell where a total bandwidth of $10 \mathrm{MHz}$ (50 RBs) [24] is uniformly allocated to $50 \mathrm{MSs}$, the number of MSs to be located simultaneously is less than or equal to 50 , due to different LCS qualities requested.

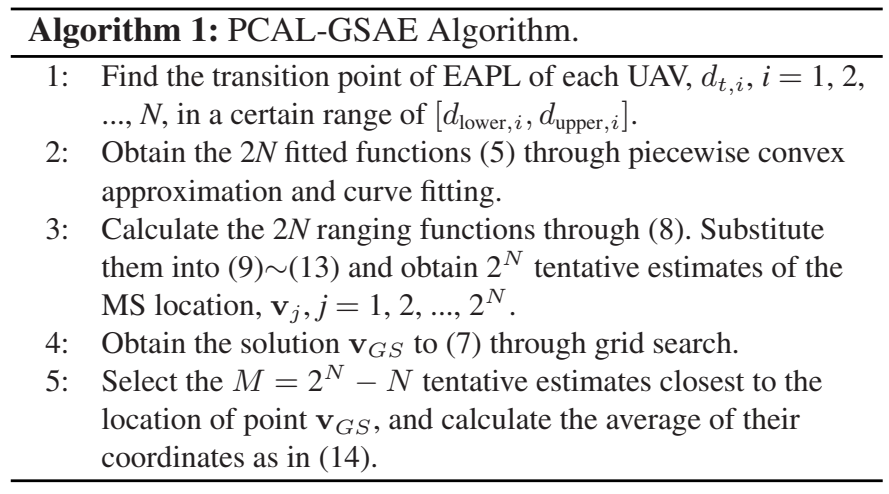

TABLE I

COMPUTATIONAL COMPLEXITY ANALYSIS. $K$-NUMBER OF EQUATIONS, $P$ - NUMBER OF VARIABLES, $\epsilon$-REQUIRED ACCURACY

\begin{tabular}{l|l|l}
\hline Algorithm & $\begin{array}{l}\text { Complexity per Itera- } \\
\text { tion }\end{array}$ & $\begin{array}{l}\text { Maximum Number of } \\
\text { Iterations }\end{array}$ \\
\cline { 1 - 1 } PCAL-GSAE & $\mathcal{O}\left(P^{2} K^{2}\right)$ & $\mathcal{O}\left(\sqrt{P} \log _{10}\left(\epsilon^{-1}\right)\right)$ \\
\cline { 1 - 1 } LSRE [6] & & \\
\cline { 1 - 1 } RSDPE [7] & & $\mathcal{O}\left(\log _{10}\left(\epsilon^{-2}\right)\right)$ \\
\cline { 1 - 2 } LSO-PLEc [19] & $\mathcal{O}\left(P^{2} K\right)$ &
\end{tabular}

\section{Cramer-Rao Lower Bound}

The CRLB on localization error is derived to evaluate the effectiveness of the proposed PCAL approach. In case of small curve fitting errors, the CRLB of localization, $\sigma_{\mathrm{CRLB}}^{2}$, is approximately an unbiased CRLB. The probability density function (PDF) of (5) distorted by shadowing is given by

$$
f_{P L_{i} \mid d_{i}}=\frac{1}{\sqrt{2 \pi} \sigma_{R S S}} e^{-\frac{\left(P L_{i}-\left(A_{i} d_{i}^{B_{i}}+\beta_{i}\right)\right)^{2}}{2 \sigma_{R S S, i}^{2}}}
$$

The Fisher information matrix (FIM), $\mathbf{F}$, for location estimate is computed as the expectation conditioned on $\mathbf{v}$, i.e., $\mathbf{F}=\mathbb{E}\left[\left(\frac{\partial \mathbf{G}}{\partial \mathbf{v}}\right)\left(\frac{\partial \mathbf{G}}{\partial \mathbf{v}}\right)^{\mathrm{T}}\right]$, where $\mathbf{G}=\left[\ln \left(f_{P L_{1} \mid d_{1}}\right), \ldots, \ln \left(f_{P L_{N} \mid d_{N}}\right)\right]$. Thus, it can be derived that

$$
\mathbf{F}=\sum_{i=1}^{N} \frac{1}{\sigma_{R S, i}^{2}}\left(A_{i} B_{i}\right)^{2} d_{i}^{2 B_{i}-4}\left(\mathbf{v}-\mathbf{w}_{i}\right)\left(\mathbf{v}-\mathbf{w}_{i}\right)^{T}
$$

Denoting $\mathbf{J}=\mathbf{F}^{-1}$ as the inverse of $\mathbf{F}$, and the CRLB is computed as the trace of $\mathbf{J}$, i.e., $\sigma_{\text {CRLB-PCAL }}^{2} \geq \operatorname{tr}[\mathbf{J}]$.

The unbiased CRLB is dependent on both fitted parameters and propagation distance, while the FIM and CRLB are independent of $S_{0, i}$ and $\beta_{i}$. Define the ratio of the CRLB of $d_{i}$ of the approaches in [6]-[8] to that of PCAL as $\varepsilon_{i}=\sigma_{\text {CRLB- } \log , d_{i}}^{2} / \sigma_{\text {CRLB-PCAL }, d_{i}}^{2}=$ $\left(A_{i}^{2} B_{i}^{2} d_{i}^{2 B_{i}} \ln ^{2} 10\right) /\left(100 \eta_{i}^{2}\right)$, where the CRLBs of [6]-[8] are same since they both apply the same path loss model. When UAV is at $h_{i}=500 \mathrm{~m}$ high, $\varepsilon_{i} \geq 38.46$ is achieved at any location within a cell, implying higher localization accuracy of PCAL compared to [6]-[8].

\section{COMPLEXITY ANALYSIS}

Table I presents the complexity analysis following the approach in [23]. The proposed PCAL-GSAE scheme requires the same order of complexity as LSRE [6] and RSDPE [7], and a higher overall complexity than LSO-PLEc [19], while achieving a significant performance gain over all of them, as shown in Section V. The complexity of GSAE is negligible compared to that of PCAL. The complexity of PCAL-GSAE 


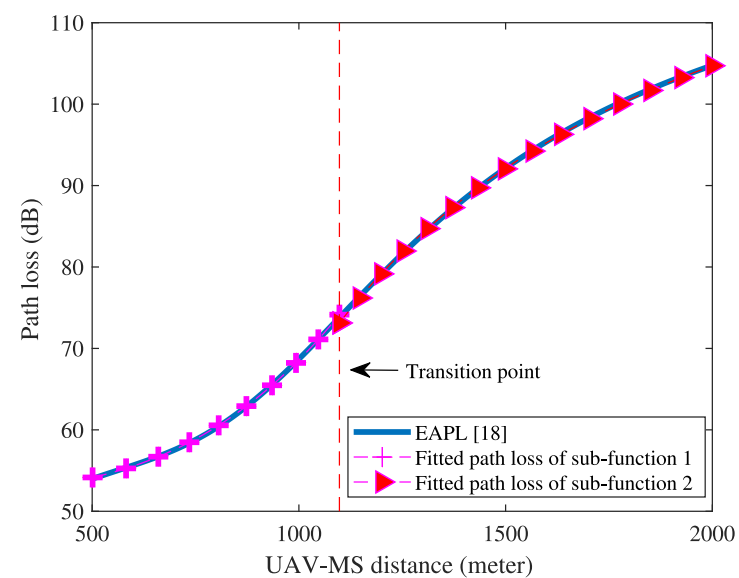

Fig. 2. Curve fitted path loss model in comparison to EAPL [18] with UAV altitudes $h_{i}=500 \mathrm{~m}(i=1, \ldots, 4)$ and cell radius $R=1000 \mathrm{~m}$.

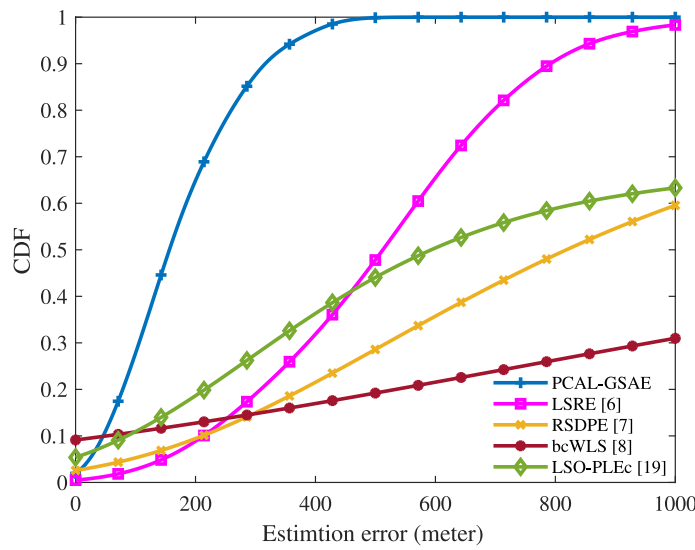

Fig. 3. CDFs of estimation error of PCAL-GASE, LSRE [6], RSDPE [7], bcWLS [8] and LSO-PLEc [19] with $N=4$ UAV-BSs and TS only.

is same as that of PCAL without ambiguity, since the $2^{N}$ tentative estimates are independent of each other.

\section{Simulation Results}

The performance of the proposed PCAL-GSAE scheme is evaluated by Monte-Carlo simulation with $N=4$ UAV-BSs supported hexagon cells. The AS standard deviation is set to $\sigma_{A S, i}=4.4 \mathrm{~dB}$ [17] in Figs. 4 and 5. Assume perfect knowledge of the EAPL parameters $a_{0}, a_{1}, b_{0}$ and $b_{1}$ given in Subsection III-A.

Fig. 2 depicts the approximated path loss in (5), compared with the original EAPL, when all UAVs' altitude is $h_{i}=500 \mathrm{~m}(i=1, \ldots, 4)$ and the cell radius is $R=1000 \mathrm{~m}$. The approximated path loss by piecewise convex approximation and curve fitting are expressed as

$$
\widehat{P L_{i}} \approx \begin{cases}5.772 \mathrm{e}-9 d_{i}^{3.15}+52.31 & d_{\mathrm{lower}, i} \leq d_{i} \leq d_{t} \\ -6.772 \mathrm{e} 4 d_{i}^{-0.9792}+144.6 & d_{t}<d_{i} \leq d_{\mathrm{upper}, i}\end{cases}
$$

where $d_{\text {lower }, i}=h_{i}$, and $d_{\text {upper }, i}=2 R$, and $d_{t, i}=1098 \mathrm{~m}$. The approximation results without the PLE knowledge match the EAPL model perfectly within $d_{i} \in\left[d_{\text {lower }, i}, d_{\text {upper }, i}\right]$.

Fig. 3 shows the cumulative density function (CDF) of PCAL-GSAE, with the same simulation setup as Fig. 2, in comparison to those of the approaches in [6]-[8] with perfectly known and equal PLEs and the LSO-PLEc approach in [19]. For fair comparison, the distance $d_{i}$ estimated by the approaches in [6]-[8] and [19] is capped at $d_{\mathrm{upper}, i}$.

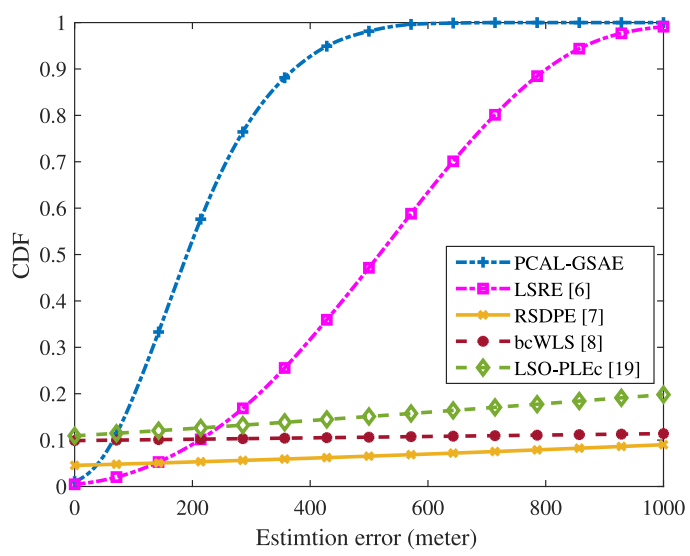

Fig. 4. CDFs of estimation error of PCAL-GASE, LSRE [6], RSDPE [7], bcWLS [8] and LSO-PLEc [19] with $N=4$ UAV-BSs, TS and AS of standard deviation $\sigma_{A S}=4.4 \mathrm{~dB}$.

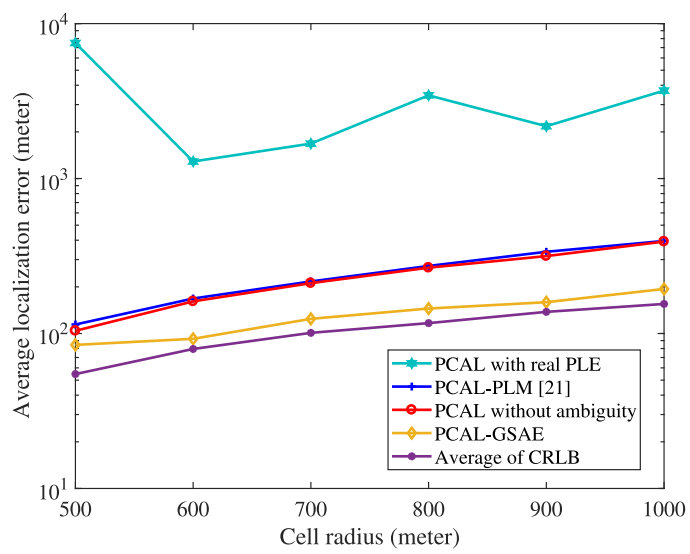

Fig. 5. Average localization error of PCAL with real PLEs, PCAL-PLM [21], PCAL without ambiguity, PCAL-GSAE, and average CRLB with $N=4 \mathrm{UAV}$ BSs, UAV altitudes $h_{i}=200 \mathrm{~m}(i=1, \ldots, 4)$, and AS of standard deviation $\sigma_{A S}=4.4 \mathrm{~dB}$.

PCAL-GSAE significantly outperforms the other approaches when the estimation error is larger than $50 \mathrm{~m}$, due to its higher robustness against TS, while an error of less than $50 \mathrm{~m}$ indicates an occasional case where ambiguity error plays a dominant role. Fig. 4 shows that with AS, the proposed PCAL-GSAE scheme maintains a performance comparable to the case without AS, while the other approaches suffer significant performance degradation compared to Fig. 3 .

Fig. 5 shows the average localization error versus cell radius, which varies from $500 \mathrm{~m}$ to $1000 \mathrm{~m}$, with UAV altitude $h_{i}=200 \mathrm{~m}(i=$ $1, \ldots, 4)$. When combined with PCAL, the proposed GSAE approach is more effective to eliminate ambiguity than the PLM approach in [21]. The performance of PCAL-GSAE is close to the CRLB derived. Also, PCAL-GSAE achieves higher accuracy than PCAL without ambiguity, because taking average of the selected tentative estimates is effective in mitigating errors caused by shadowing. PCAL with real PLE values demonstrates a much worse performance than PCAL with unknown PLEs, since the approximations in (5) and (6) and GSAE are disabled under perfect knowledge of PLEs, which makes the algorithm more vulnerable to shadowing.

\section{CONCLUSION}

We have proposed a PCAL scheme for multiple UAV-BSs assisted and RSS based MS localization without requiring PLEs to be equal and 
perfectly known. The two-step approximations by piecewise convex approximation and curve fitting yield a convex localization problem that matches the EAPL model [18] very well. The localization problem is then converted to linear via TSE which can be solved by SDP with equivalent complexity as [6] and [7]. The proposed PCAL approach with unknown and unequal PLEs significantly outperforms the approaches in [6]-[8] with perfectly known and equal PLEs, and [19] with unknown and unequal PLEs, demonstrating higher robustness against shadowing especially AS in the scenario of UAV. The proposed GSAE method can eliminate ambiguity more effectively than the PLM method in [21]. The effect of UAV trajectory design on localization will be considered in our future work.

\section{REFERENCES}

[1] M. Alzenad, A. El-Keyi, F. Lagum, and H. Yanikomeroglu, "3-D placement of an unmanned aerial vehicle base station (UAV-BS) for energyefficient maximal coverage," IEEE Wireless Commun. Lett., vol. 6, no. 4, pp. 434-437, Aug. 2017.

[2] A. Merwaday and I. Guvenc, "UAV assisted heterogeneous networks for public safety communications," in Proc. IEEE Wireless Commun. Netw. Conf. Workshops, New Orleans, LA, USA, Mar. 2015, pp. 329-334.

[3] C. Luo, S. I. McClean, G. Parr, L. Teacy, and R. D. Nardi, "UAV position estimation and collision avoidance using the extended Kalman filter," IEEE Trans. Veh. Technol., vol. 62, no. 6, pp. 2749-2162, Jul. 2013.

[4] R. M. Vaghefi, M. R. Gholami, R. M. Buehrer, and E. G. Strom, "Cooperative received signal strength-based sensor localization with unknown transmit powers," IEEE Trans. Signal Process., vol. 61, no. 6, pp. 13891403, Mar. 2013.

[5] S. Tomic, M. Beko, and R. Dinis, "RSS-based localization in sensor networks using convex relaxation: Noncooperative and cooperative schemes," IEEE Trans. Veh. Technol., vol. 64, no. 5, pp. 2037-2050, May 2015.

[6] Z. Wang, H. Zhang, T. Lu, and T. A. Gulliver, "Cooperative RSS-based localization in wireless sensor networks using relative error estimation and semidefinite programming," IEEE Trans. Veh. Technol., vol. 68, no. 1, pp. 483-497, Jan. 2019.

[7] Y. Hu and G. Leus, "Robust differential received signal strength-based localization,” IEEE Trans. Signal Process., vol. 65, no. 12, pp. 3261-3276, Jul. 2017.

[8] V. Kumar, R. Arablouei, R. Jurdak, B. Kusy, and N. W. Bergmann, "RSSI-based self-localization with perturbed anchor positions," in Proc. IEEE 28th Int. Symp. Pers., Indoor Mobile Radio Commun., Montreal, QC, Canada, Oct. 2017, pp. 1-6.

[9] S. Mazuelas, R. M. Lorenzo, A. Bahillo, P. Fernandez, J. Prieto, and E. J. Abril, "Topology assessment provided by weighted barycentric parameters in harsh environment wireless location systems," IEEE Trans. Signal Process., vol. 58, no. 7, pp. 3842-3857, Jul. 2010.
[10] J. Prieto, S. Mazuelas, A. Bahillo, P. Fernandez, R. M. Lorenzo, and E. J. Abril, "Adaptive data fusion for wireless localization in harsh environments," IEEE Trans. Signal Process., vol. 60, no. 4, pp. 1585-1596, Apr. 2012.

[11] S. Fang, Y. Hsu, B. Lu, and W. Kuo, "A calibration-free RSS-based mobile positioning system," in Proc. IEEE Veh. Technol. Conf.-Spring, Yokohama, Japan, May 2012, pp. 1-5.

[12] L. Cheng, C. Wu, Y. Zhang, and Y. Wang, "An indoor localization strategy for a mini-UAV in the presence of obstacles," Int. J. Adv. Robot. Syst., vol. 9 , no. 4, pp. 1-8, Oct. 2012

[13] I. Ahmad, N. W. Bergmann, R. Jurdak, and B. Kusy, "Towards probabilistic localization using airborne mobile anchors," in Proc. IEEE Int. Conf. Pervasive Comput. Commun. Workshops, Sydney, NSW, Australia, Mar. 2016, pp. 1-4.

[14] D. W. Matolak and R. Sun, "Air-ground channel characterization for unmanned aircraft systems - Part I: Methods, measurements, and models for over-water settings," IEEE Trans. Veh. Technol., vol. 66, no. 1, pp. 26-44, Jan. 2017.

[15] K. Wang et al., "Path loss measurement and modeling for low-altitude UAV access channels," in Proc. IEEE VTC-Fall, Toronto, ON, Canada, Sep. 2017, pp. 1-5.

[16] A. Al-Hourani, and K. Gomez, "Modeling cellular-to-UAV path-loss for suburban environments," IEEE Wireless Commun. Lett., vol. 7, no. 1, pp. 82-85, Feb. 2018.

[17] D. W. Matolak, R. Sun, and W. Rayess, "Air-ground channel characterization for unmanned aircraft systems-Part IV: Airframe shadowing," IEEE Trans. Veh. Technol., vol. 66, no. 9, pp. 7643-7652, Sep. 2017.

[18] H. Sallouha, M. M. Azari, A. Chiumento, and S. Pollin, "Aerial anchors positioning for reliable RSS-based outdoor localization in urban environments," IEEE Wireless Commun. Lett., vol. 7, no. 3, pp. 376-379, Jun. 2018.

[19] S. Mazuelas et al., "Robust indoor positioning provided by real-time RSSI values in unmodified WLAN networks," IEEE J. Sel. Topics Signal Process., vol. 3, no. 5, pp. 821-831, Oct. 2009.

[20] M. Hasanzade, O. Herekoglu, N. K. Ure, E. Koyuncu, R. Yeniceri, and G. Inalhan, "Localization and tracking of RF emitting targets with multiple unmanned aerial vehicles in large scale environments with uncertain transmitter power," in Proc. Int. Conf. Unmanned Aircr. Syst., Miami, FL, USA, Jul. 2017, pp. 1058-1065.

[21] S. Boyd and L. Vandenberghe, Convex Optimization. Cambridge, U.K.: Cambridge Univ. Press, 2004.

[22] M. D. Buhmann and A. Iserles, Approximation Theory and Optimization. Cambridge, U.K.: Cambridge Univ. Press, 1997.

[23] L. A. Vandenberghe and S. B. Boyd, "Semidefinite programming," SIAM Rev., vol. 38, no. 1, pp. 49-95, Mar. 1996.

[24] 3GPP Specification Release 15. [Online]. Available: https://www.3gpp. org/DynaReport/SpecReleaseMatrix.htm 